\title{
Signaling at A-Kinase Anchoring Proteins Organizes Anesthesia-Sensitive Memory in Drosophila
}

\author{
Martin Schwaerzel, Andrea Jaeckel, and Uli Mueller \\ Saarland University, Faculty 8.3 Life Science, Department of Zoology and Physiology, 66041 Saarbrucken, Germany
}

The ubiquitous cAMP-protein kinase A (PKA) signaling pathway exhibits complex temporal requirements during the time course of associative memory processing. This directly raises questions about the molecular mechanisms that provide signaling specificity to this pathway. Here, we use Drosophila olfactory conditioning to show that divergent cAMP signaling is mediated by functionally distinct pools of PKA. One particular pool is organized via the PKA regulatory type II subunit at the level of A-kinase anchoring proteins (AKAPs), a family of scaffolding proteins that provides focal points of spatiotemporal signal integration. This AKAP-bound pool of PKA is acting within neurons of the mushroom bodies to support a late phase of aversive memory. The requirement for AKAP-bound PKA signaling is limited to aversive memory, but dispensable during appetitive memory. This finding suggests the existence of additional mechanisms to support divergence within the cAMP-PKA signaling pathway during memory processing. Together, our results show that subcellular organization of signaling components plays a key role in memory processing.

Key words: protein kinase A; memory phases; A kinase anchoring protein; Drosophila; camp; learning; memory

\section{Introduction}

The cellular and molecular mechanisms underlying induction and maintenance of memory are highly dynamic and comprise distinct phases such as anesthesia-sensitive forms of short-term (STM) and midterm memory (MTM), as well as anesthesiaresistant forms, like anesthesia-resistant memory (ARM) and long-term memory (LTM), which have been experimentally distinguished in all species (DeZazzo and Tully, 1995; Abel and Lattal, 2001; Kandel, 2001). In Drosophila olfactory learning, mutants along the cAMP-protein kinase A (PKA) pathway affect different memory phases; whereas mutation of the adenylyl cyclase rutabaga or the cAMP-specific phosphodiesterase dunce affects STM and subsequent memory phases, mutation of PKA catalytic subunit (DCO) affects MTM. LTM in Drosophila, as in other species, requires PKA-dependent phosphorylation of CREB (cAMP response element-binding protein) transcription factors (Margulies et al., 2005; McGuire et al., 2005; Skoulakis and Grammenoudi, 2006). This differential requirement of the cAMP-PKA cascade in memory processing directly raises questions about the molecular mechanisms that provide specificity to this ubiquitous signaling pathway.

A-kinase anchoring proteins (AKAPs) provide platforms for the assembling of multiprotein complexes, where numerous signaling enzymes become organized within the spatiotemporal do-

Received 0ct. 25, 2006; revised Dec. 25, 2006; accepted Dec. 26, 2006.

The Drosophila Genomic Resource Center (Indiana University Bloomington, Bloomington, IN) provided us with Terrence Murphey's Gateway collection of expression vectors. We thank Angelika Gardezi, Michael Glander, and Jens Wiegant for excellent technical assistance. Björn Brembs and Hiromu Tanimoto provided helpful comments on this manuscript.

Correspondence should be addressed to Martin Schwaerzel, Saarland University, Department of Zoology and Physiology, P.0. Box 151150, 66041 Saarbrucken, Germany. E-mail:m.schwaerze@@mx.uni-saarland.de.

DOI:10.1523/JNEUROSCI.4622-06.2007

Copyright $\odot 2007$ Society for Neuroscience $\quad$ 0270-6474/07/271229-05\$15.00/0 main. Whereas cellular studies have long treated this mechanism as a key concept for providing the cAMP-PKA pathway with signaling specificity (Colledge et al., 2000; Wong and Scott, 2004; Hoshi et al., 2005), we here explore its in vivo aspect during processing of associative memories in Drosophila. The PKA holoenzyme is composed of regulatory and catalytic subunits, respectively. Anchoring to AKAP complexes is mediated by PKA regulatory subunits with regulatory type I (RI) and RII using different molecular mechanisms for this interaction. In particular, the molecular mechanism underlying anchoring of PKA via the RII subunit (PKA-RII) to AKAP complexes is highly conserved across phyla and dependent on interaction between the N-terminal docking domain of PKA-RII with an amphipatic helix of AKAP molecules (Hausken et al., 1994; Colledge and Scott, 1999). Exploiting this stereotyped interaction, we adapted these findings to the in vivo level and chose an approach that allows us to test for per se involvement of AKAP-bound PKA: the endogenous competitor of PKA-RII (eCOPR2 peptide) constitutes the docking domain of Drosophila PKA-RII and shall thus compete with endogenous full-length PKA-RII for AKAP-binding sites without interfering with cAMP or PKA catalytic function. To control for eCOPR2 specificity, we deleted 13 amino acids essential for function of the docking domain in a control peptide, eCOPR2 $\Delta$ (Hausken et al., 1994). This manipulation is supposed to be specific for PKA-RII-mediated anchoring without disturbing PKA-RI-anchored or soluble PKA pools.

Olfactory conditioning is a well established paradigm for investigating the molecular and cellular substrates underlying associative memory processing (Gerber et al., 2004; McGuire et al., 2005). Drosophila genetic techniques allow targeting expression of the eCOPR2 competitor peptide in various neuropils associated with olfactory memory and, thus, make it possible to probe the impact of AKAP-bound PKA-RII signaling on associative 
memory. Moreover, the paradigm allows for a direct comparison between cAMP-dependent aversive and appetitive conditioning and, thus, gives insight into the molecular mechanisms underlying their internal organization, on both the molecular and neuronal network levels (Schwaerzel et al., 2003).

\section{Materials and Methods}

Fly care and behavioral experiments. All flies were raised at $24^{\circ} \mathrm{C}$ and $60 \%$ relative humidity with a $14 / 10 \mathrm{~h}$ light/dark cycle on cornmeal-based food according to the Würzburg recipe (Guo et al., 1996). Genotypes including the temperature-sensitive galactosidase $80\left(\mathrm{Gal} 80^{\mathrm{ts}}\right)$ repressor (McGuire et al., 2003) were raised at the restrictive temperature $\left(19^{\circ} \mathrm{C}\right)$. For inactivation of the $\mathrm{Gal} 80^{\text {ts }}$ repressor, flies were placed at $30^{\circ} \mathrm{C}$ for at least $14 \mathrm{~h}$ and immediately tested for memory performance. Application of cold amnestic treatment $(1 \mathrm{~min})$ was performed in small plastic vials precooled on ice (Tempel et al., 1983). In general, all experimental flies were transferred to fresh food vials for up to $48 \mathrm{~h}$ before the test and starved for $18 \mathrm{~h}$ in empty vials equipped with moist filter paper to prevent desiccation. Behavioral experiments were performed in dim red light at $70 \%$ relative humidity as described previously (Schwaerzel et al., 2003).

Generation of transgenic flies. For constructing the upstream activating sequence (UAS)-expression vectors containing the competitor peptides UAS-eCOPR2 and UAS-eCOPR2 $\Delta$ we amplified the $\mathrm{N}$-terminal part of the endogenous Drosophila RII subunit (Park et al., 2000) from wild-type Canton-S genomic DNA using proof reading PCR. The thermal cycling conditions were set at $95^{\circ} \mathrm{C}$ for $5 \mathrm{~min}$, followed by 35 cycles of $30 \mathrm{~s}$ denaturation, $30 \mathrm{~s}$ of annealing at $60^{\circ} \mathrm{C}$, and $60 \mathrm{~s}$ of elongation at $72^{\circ} \mathrm{C}$. Final elongation was set at $72^{\circ} \mathrm{C}$ for $5 \mathrm{~min}$. The forward primers aligned within the open reading frame including consensus sequences to be used with the Gateway vectors (UAS-eCOPR2, C ACC ATG TCG AGC GAT TCG AGT CGA AGG), or deleted the first 13-most N-terminal amino acids from the transcript in case of UAS-eCOPR2 $\Delta$ (C ACC ATG GAG GAG CTG AAG GAG GTG). In both cases, a common reverse primer (TGA GTT GAC GCT CAG CTG GTC ATC) was used. The respective PCR products were cloned into pENTR-D TOPO (Invitrogen, Eugene, OR) and further cloned into the pUAST Drosophila transfection vector from Terrence Murphy's Gateway vector collection (Drosophila Genomics Resource Center, Indiana University Bloomington, Bloomington, IN) following manufacturer's instructions (Invitrogen). Germ-line transformation was done by standard procedure using an isogenized Drosophila $\mathrm{w}^{1118}$ strain out-crossed against Canton-S for 70 generations.

Statistical analysis. Comparison was performance using one-way ANOVA with a post hoc test including the Bonferroni correction for multiple comparisons.

\section{Results}

AKAP-bound PKA-RII supports a distinct memory phase after aversive training

To investigate the role of AKAP-bound PKA-RII in associative memory processing, we expressed the competitor peptide throughout the Drosophila nervous system under control of the pan-neuronal elav Gal4-driver (Fig. 1A). Performance of elavGal4/UAS-eCOPR2 flies was characterized by wild-type-like memory performance for up to $60 \mathrm{~min}$ ( $p>0.05$ when compared with genetic controls), followed by accelerated memory loss between 60 and $120 \mathrm{~min}$. Finally, memory runs down into a stable plateau about half the level of the performance observed in control animals at $2-3 \mathrm{~h}$ after training $(p<0.01)$. Genetic controls bearing either the Gal4 or the UAS-eCOPR2 element alone (elav-Gal4/+ and UAS-eCOPR2/+, respectively) showed wildtype-like performance, as did flies expressing the truncated peptide unable to compete with endogenous PKA-RII for AKAP binding (elav-Gal4/UAS-eCOPR2 $\Delta$ ). This result leads to the conclusion that signaling via AKAP-bound PKA-RII is not required to support overall associative processes, as memory is initially acquired to a normal extent. Rather, AKAP-bound PKA-RII sup-

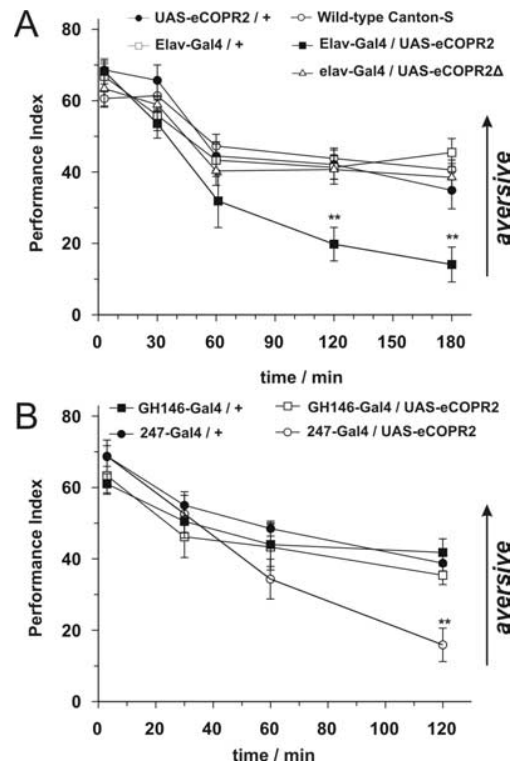

Figure 1. AKAP-bound PKA-RII supports a distinct phase of aversive olfactory memory. Anchoring PKA-RII to AKAP complexes can be interfered with by ectopic expression of the UASeCOPR2 peptide which constitutes the docking domain of PKA-RII and, thus, dislodges endogenous PKA-RII from AKAP complexes. $A$, Pan-neuronal expression of the competitor (elav-Gal4/ UAS-eCOPR2) results in altered memory kinetics: although memory is acquired to a wild-type extent, an accelerated loss of memory becomes obvious between 60 and 120 min after training, followed by a stable plateau at about half the level of wild-type-like performance at 120-180 $\min$ ( $p>0.05$ for time-points up to $60 \mathrm{~min} ; p<0.01$ at 120 and $180 \mathrm{~min}$ ). $\boldsymbol{B}$, AKAP-bound PKA-RII is required within the mushroom bodies (247-Gal4/UAS-eCOPR2) to support aversive memory processing. The behavioral defect is indistinguishable from pan-neuronal expression ( $p<0.01$ at 120 min; when comparing 247-Gal4/UAS-eCOPR2 to elav-Gal4/UAS-eCOPR2: $p$ values $>0.05$ at all time points). All data represent means \pm SEM; $n=6$ for all groups, except for wild-type $n=9 .{ }^{*} p<0.05 ;{ }^{* *} p<0.01$.

ports a distinct memory phase after aversive conditioning with a behavioral defect becoming obvious within a time window $1-2 \mathrm{~h}$ after training.

\section{AKAP-bound PKA-RII-dependent memory is localized to the mushroom bodies}

In an attempt to localize the neuronal site(s) within the Drosophila brain where AKAP-bound PKA-RII is required to support the particular memory phase after aversive training, we spatially restricted expression of the UAS-eCOPR2 peptide to different neuropils associated with olfactory information processing (Fig. $1 B$ ). The $247-G a l 4$ driver addresses $\sim 700$ cells within the mushroom bodies (MBs), a structure that supports acquisition of olfactory memories via a cAMP-dependent process of synaptic plasticity as revealed by local rescue of the learning mutant rutabaga (Zars et al., 2000; Schwaerzel et al., 2002). Expressing the competitor peptide within those cells results in accelerated memory decline indistinguishable from the effect observed in the case of panneuronal expression ( $p<0.01$ when comparing 247-Gal4/UASeCOPR2 to genetic controls; $p$ values $>0.05$ when comparing between 247-Gal4/UAS-eCOPR2 and elav-Gal4/UAS-eCOPR2 from Fig. $1 \mathrm{~A}$ ). On the contrary, targeting the peptide to the projection neurons (GH146-Gal4/UAS-eCOPR2), which provide the major route of olfactory input to the MBs, had no detrimental effect on memory performance.

To distinguish between effects caused by acute AKAP-bound PKA-RII signaling and unspecific developmental defects that might be caused by the competitor peptide, we restricted expres- 


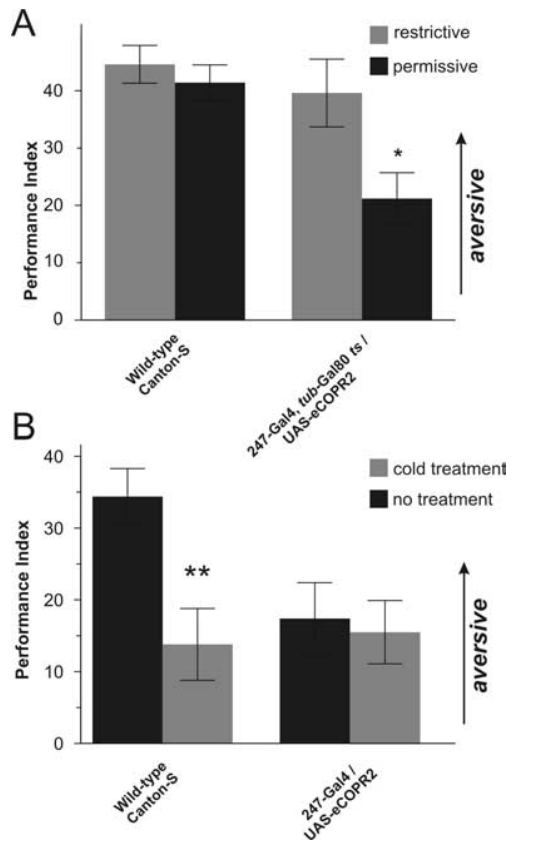

Figure 2. AKAP-bound PKA-RII acutely supports a distinct form of anesthesia-sensitive memory. $A$, Acute induction of the competitor peptide by use of the conditional blocker GAL $80^{\text {ts }}$ produces loss of $3 \mathrm{~h}$ memory performance equal to permanent expression ( $p<0.05$ when comparing permissive to restrictive conditions; $p>0.05$ when comparing performance of 247-Gal4, tub-Gal80ts/UAS-eCOPR2 flies under permissive conditions to elav-Gal4/UASeCOPR2 from Fig. 1 A). $\boldsymbol{B}$, Three hour memory is composed of two separable memory phases, ASM and ARM. Cold amnestic treatment leads to a significant decline of memory performance in wild-type flies, revealing (ARM, gray bars) and ARM plus ASM (black bars). In 247-Gal4/UASeCOPR2 flies, ASM is completely abolished regardless of amnestic treatment ( $p>0.05)$. All data represent means $\pm \mathrm{SEM} ; n=6$ for all groups, except for wild-type $n=8 .{ }^{*} p<0.05$; ${ }^{* *} p<0.01$.

sion of UAS-eCOPR2 in the temporal domain to the adult stage (Fig. 2A). When expression is induced in the MBs exclusively within the adult stage $18 \mathrm{~h}$ before behavioral testing (McGuire et al., 2003), flies display poor memory performance at $3 \mathrm{~h}$, indistinguishable from permanent peptide expression $(p<0.05$, when comparing permissive and restrictive conditions in 247Gal4, tub-Gal80ts/UAS-eCOPR2; $p>0.05$ when compared with 247-Gal4/UAS-eCOPR2 from Fig. $1 B$ ). Thus, AKAP-bound PKA-RII is acutely required within the MBs (i.e., within the same structure that acquires short-term memory via a cAMPdependent mechanism in the first place) (Zars et al., 2000; Schwaerzel et al., 2002, 2003).

\section{AKAP-bound PKA-RII supports a late phase of anesthesia- sensitive aversive memory}

After interference with AKAP-bound PKA-RII signaling, a residual memory is still observable and remains stable without additional decline for $2-3 \mathrm{~h}$ after aversive training (Fig. $1 A, B$ ). To further characterize this residual memory's performance, we applied cold amnestic treatment to erase all anesthesia-sensitive forms of olfactory memory (Quinn and Dudai, 1976). In wildtype flies, this treatment results in a significant decline of $3 \mathrm{~h}$ memory compared with untreated controls (Fig. $2 B$ ). Thus, the observed memory is composed of two separable phases, anesthesia-sensitive memory (ASM), which is lost after cooling, and ARM, which remains in the cold-treated group. On the contrary, flies expressing the competitor peptide in the MBs only perform according to ARM, as indicated by equally low levels of performance regardless of cooling ( $p$ values $>0.05$ when com-
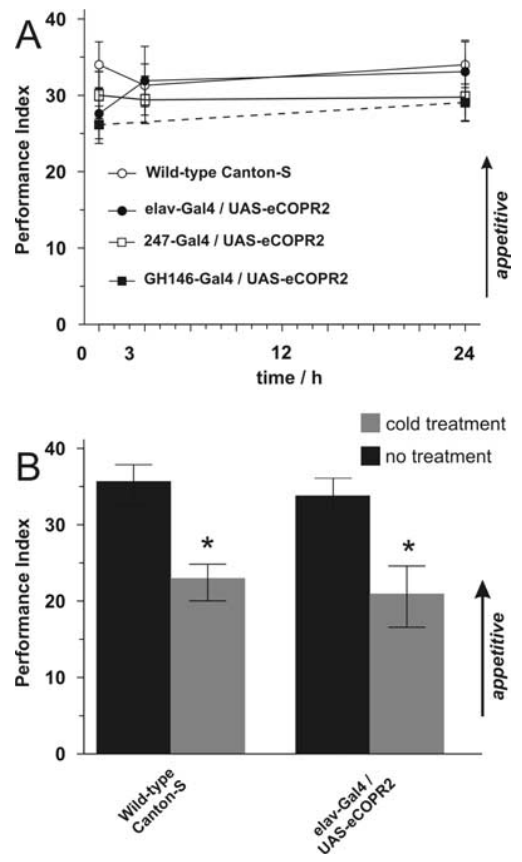

Figure 3. Appetitive and aversive memories are dissociated on the level of AKAP-bound PKA-R II signaling. Sugar reward leads to CAMP-dependent formation of appetitive memory. $\boldsymbol{A}$, AKAP-bound PKA-R II is not required for performance of appetitive memory, as revealed by wild-type-like performance of flies expressing the competitor in various neuropils. Neither pan-neuronal (elav-Gal4/UAS-eCOPR2), nor MB-specific (247-Gal4/UAS-eCOPR2) or projection neuron-specific expression (GH146-Gal4/UAS-eCOPR2) impaired memory performance after appetitive training for up to $24 \mathrm{~h}$ ( $p$ values $>0.05$ ). $\boldsymbol{B}$, When cold-amnestic treatment is applied 30 min before testing $3 \mathrm{~h}$ memory, wild-type flies show a significant decline in performance when comparing treated (gray bars) to nontreated controls (black bars; $p<0.05$ ). Pan-neuronal expression of the competitor in elav-Gal4/UAS-eCOPR2 flies shows memory performance indistinguishable from wild-type controls ( $p$ values $>0.05$ ). All data represent means \pm SEM; $n=6$ for all groups, except for wild-type $n=9 .{ }^{*} p<0.05 ;{ }^{* *} p<0.01$.

paring treated and untreated groups of 247-Gal4/UAS-eCOPR2 flies, or alternatively comparing them to treated wild-type animals). This experiment separates ASM into two functionally distinct phases: early and late AMS. The latter requires AKAPbound PKA-RII signaling in the MBs as revealed by its loss in the competitor-expressing group.

AKAP-bound PKA-RII is "bypassed" in appetitive memory Depending on the reinforcing stimulus used during the training procedure, either aversive or appetitive memories can be acquired. Both types of short-term memory are localized to the MBs and acquired by a cAMP-dependent mechanism of plasticity (Schwaerzel et al., 2003). Downstream of cAMP signaling, however, AKAP-bound PKA-RII is dispensable for performance of appetitive memory (Fig. $3 A$ ): neither pan-neuronal nor restricted expression of the UAS-eCOPR2 peptide within the various neuropils involved in processing olfactory information produced any significant memory decline for up to $24 \mathrm{~h}$ ( $p$ values $>0.05$ for any time point tested). However, appetitive memory, like aversive memory, is composed of ASM and ARM (Fig. $3 B$ ) as revealed by the significant decline in wild-type animals after cold anesthesia $(p<0.05)$. A similar decline in memory performance is observed in flies expressing the competitor peptide throughout the nervous system; this suggests that appetitive ASM is independent of AKAP-bound PKA-RII. 


\section{Discussion}

Here, we pioneered the role of AKAPs as organizers of PKA signaling in Drosophila associative olfactory memory processing. First, we showed that after aversive conditioning ASM can be separated into two phases; whereas an early phase of ASM is AKAP-independent, late ASM requires AKAP-bound PKA-RII signaling within the MBs. Second, we showed that, after appetitive conditioning, ASM "bypasses" this AKAP-bound pool of PKA. This shows that PKA signaling at AKAP complexes does not disrupt associative functions per se, but that functionally distinct pools of PKA must exist on a subcellular level to serve defined functions during memory processing. We suggest a functional model in which cAMP signaling is distributed on the subcellular level by involvement of AKAP-bound pools of PKA.

\section{AKAPs as organizing elements: dynamic processing of} anesthesia-sensitive memory after aversive conditioning

Mutants affecting different steps along the cAMP-PKA cascade have identified distinct anesthesia-sensitive memory phases in Drosophila olfactory conditioning. Mutants directly affecting cAMP (e.g., the adenylyl cyclase rutabaga or the cAMP-specific phosphodiesterase dunce) disturb formation of ASM. Further downstream within the pathway, a conditional allele of the PKA catalytic subunit DC0 X4 impairs memory performance around $20 \mathrm{~min}$ to $2 \mathrm{~h}$ after training (Li et al., 1996). The amnesiac mutant has revealed a critical time window shortly after conditioning, when olfactory memory requires activity of the dorsal paired medial (DPM) neurons to act onto the mushroom body structure to stabilize ASM. In fact, it has been this temporal requirement for Rutabaga, Dunce, DC0 X4, and Amnesiac that lead to the separation of ASM into STM and MTM. Our analysis now revealed that MTM can be further dissected into an early and a late phase. Although the early phase of MTM requires PKA signaling as revealed by the DC0 X4 allele, we have shown that it is independent of AKAP binding. However, a late phase of MTM requires AKAPbound PKA-RII signaling and supports memory performance from $60 \mathrm{~min}$ after conditioning on. As performance of this late MTM becomes completely abolished, we conclude that no redundancy exists on the molecular level to support this particular late phase. Moreover, early and late MTM must be supported by distinct pools of PKA that serve separable functions during the time course of processing.

The functional dissection of PKA into AKAP-bound and nonbound pools suggested here is derived from the eCOPR2 peptide that constitutes the AKAP binding site of endogenous Drosophila PKA-RII. Based on the conserved mechanism of this interaction across phyla, we speculate that eCOPR2 disturbs the binding of PKA-RII to all potential AKAPs. Additional experiments are now required to (1) identify the particular $\mathrm{AKAP}(\mathrm{s})$ that are involved in late MTM stabilization, and (2) to formally prove that eCOPR2 does indeed disrupt interaction between AKAPs and PKA-RII. However, as we show that eCOPR2 expression affects very specific aspects of aversive ASM processing rather than abolishing associative functions per se provides strong arguments in favor of distinct PKA pools. A previous study in mammals has shown similar impairment of memory several hours after conditioning when using microinfusion of $\mathrm{Ht}-31$, a specific competitor of PKA-RII-AKAP interaction (Moita et al., 2002). In Drosophila we could localize the effects of AKAP-bound PKA-RII on aversive ASM stabilization to 700 neurons of the MBs (i.e., to the same neurons that acquire aversive memory via a Rutabaga-dependent process of synaptic plasticity in the first place). We speculate that

\section{A aversive conditioning}

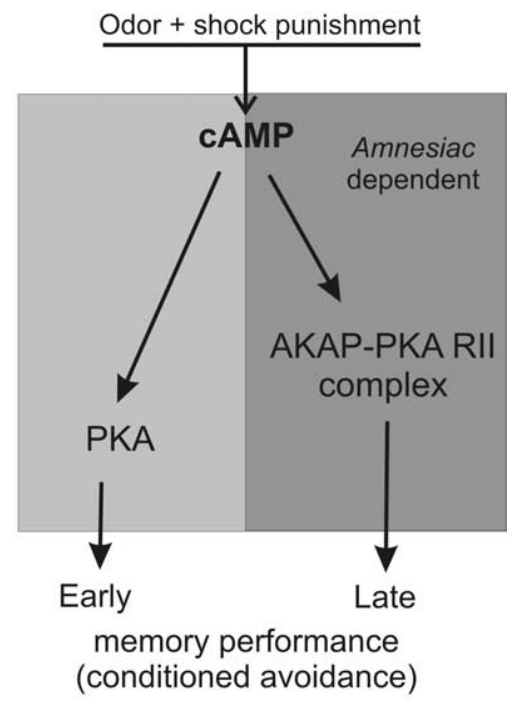

B appetitive conditioning

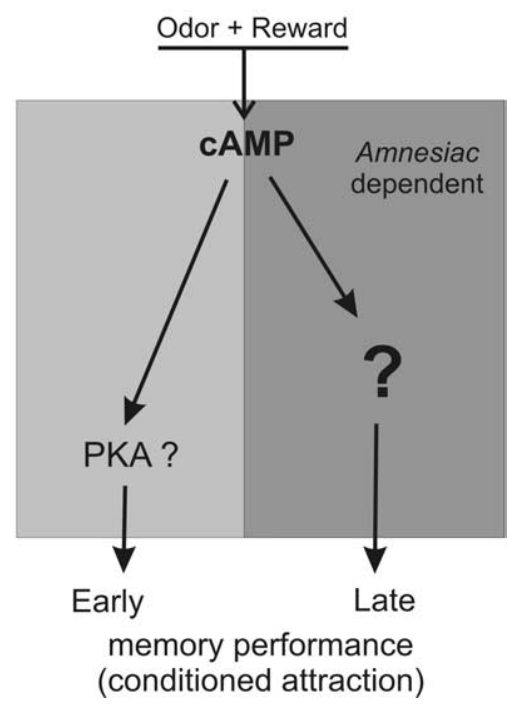

Figure 4. Model of CAMP signaling divergence in olfactory memory processing. Aversive and appetitive olfactory memories are processed within the mushroom bodies (gray box). In addition to dissociation on the level of catecholamine transmitters, these two forms of behavioral plasticity display a high degree of similarity: both memories require cAMP signaling within the mushroom bodies for proper processing, and the amnesiac mutation distinguishes between an early and a late memory phase, respectively. Here, we have shown that the late phase is dissociated on the molecular level by involvement of AKAP-PKA-RII complexes. $A$, In case of aversive memory, AKAP-bound PKA-R II signals are exclusively required to sustain later phases of memory performance. A different PKA pool is necessary for performance at early stages. $\boldsymbol{B}$, Appetitive memory is independent of the AKAP anchored pool of PKA-RIl and requires a yet unknown molecular mechanism.

processing aversive ASM from STM into early and late MTM takes place within these neurons.

In mammalian cell culture it has been shown that AKAPs are localized to specific subcellular foci of signal integration, including synaptic vesicles, ionotropic and metabotropic receptors, the cytoskeleton, or cellular organelles (Wong and Scott, 2004). Dependent on this localization, AKAPs can organize PKA signaling by selecting specific substrates to the AKAP complex. These findings can now guide research in identifying particular substrates that are involved in Drosophila aversive MTM processing. 


\section{Different routes of memory processing in aversive and appetitive ASM: comparing molecular versus neuronal "bypasses"}

Experiencing sugar reward or electric shock punishment during the training procedure gives rise to the formation of either appetitive or aversive olfactory memories. Both are dissociated on the level of catecholamines required, but display numerous similarities with respect to the neural and molecular networks involved in aversive and appetitive memory: both types of memory are acquired within the same subset of $700 \mathrm{MB}$ neurons via a Rutabaga-dependent mechanism of cAMP-mediated plasticity (Schwaerzel et al., 2003). Moreover, secretion from DPM neurons onto the MBs is required within $30 \mathrm{~min}$ after conditioning to support stabilization of aversive and appetitive ASM, respectively (Yu et al., 2005; Keene et al., 2006). However, here we showed that AKAP-bound PKA-RII dissociates between both forms, because neither pattern of competitor peptide expression affects appetitive ASM. This argues in favor of the hypothesis that different CAMP-dependent mechanisms support aversive and appetitive ASM; the remarkable stability of appetitive memory within the temporal domain (Fig. 3A) strongly supports this hypothesis. Because the eCOPR2 peptide is believed to specifically block interactions between PKA-RII and appropriate AKAPs, additional pools of PKA might support appetitive ASM processing. Additional experiments can now probe soluble PKA pools or alternative anchoring mechanisms to AKAPs via the PKA regulatory subunit type I (Banky et al., 1998).

\section{Divergent cAMP signaling in Drosophila ASM processing: a mechanistic model}

The finding that cAMP-signaling couples divergently to alternative downstream partners is a theme commonly observed throughout the animal kingdom (Muller and Carew, 1998; Sherff and Carew, 2004; Reisner et al., 2006). In Aplysia neurons, the temporal pattern of receptor activation is sufficient to trigger different cellular responses by threshold-dependent sorting mechanisms. Similar mechanisms might be in place in Drosophila olfactory conditioning, when either catecholamine receptors and/or DPM activity have the potential to trigger different threshold-dependent mechanisms of subcellular signal divergence within the MBs (Fig. 4). In Drosophila, aversive olfactory conditioning dopaminergic and DPM signals exhibit complex temporal patterns that sustain the conditioning period (Riemensperger et al., 2005; Yu et al., 2005). However, additional experiments will be required to substantiate this hypothesis.

\section{References}

Abel T, Lattal KM (2001) Molecular mechanisms of memory acquisition, consolidation and retrieval. Curr Opin Neurobiol 11:180-187.

Banky P, Huang LJ, Taylor SS (1998) Dimerization/docking domain of the type Ialpha regulatory subunit of cAMP-dependent protein kinase. Requirements for dimerization and docking are distinct but overlapping. J Biol Chem 273:35048-35055.

Colledge M, Scott JD (1999) AKAPs: from structure to function. Trends Cell Biol 9:216-221.

Colledge M, Dean RA, Scott GK, Langeberg LK, Huganir RL, Scott JD (2000) Targeting of PKA to glutamate receptors through a MAGUK-AKAP complex. Neuron 27:107-119.
DeZazzo J, Tully T (1995) Dissection of memory formation: from behavioral pharmacology to molecular genetics. Trends Neurosci 18:212-218.

Gerber B, Tanimoto H, Heisenberg M (2004) An engram found? Evaluating the evidence from fruit flies. Curr Opin Neurobiol 14:737-744.

Guo A, Li L, Xia SZ, Feng CH, Wolf R, Heisenberg M (1996) Conditioned visual flight orientation in Drosophila: dependence on age, practice, and diet. Learn Mem 3:49-59.

Hausken ZE, Coghlan VM, Hastings CA, Reimann EM, Scott JD (1994) Type II regulatory subunit (RII) of the cAMP-dependent protein kinase interaction with A-kinase anchor proteins requires isoleucines 3 and 5. J Biol Chem 269:24245-24251.

Hoshi N, Langeberg LK, Scott JD (2005) Distinct enzyme combinations in AKAP signalling complexes permit functional diversity. Nat Cell Biol 7:1066-1073

Kandel ER (2001) The molecular biology of memory storage: a dialogue between genes and synapses. Science 294:1030-1038.

Keene AC, Krashes MJ, Leung B, Bernard JA, Waddell S (2006) Drosophila dorsal paired medial neurons provide a general mechanism for memory consolidation. Curr Biol 16:1524-1530.

Li W, Tully T, Kalderon D (1996) Effects of a conditional Drosophila PKA mutant on olfactory learning and memory. Learn Mem 2:320-333.

Margulies C, Tully T, Dubnau J (2005) Deconstructing memory in Drosophila. Curr Biol 15:R700-R713.

McGuire SE, Le PT, Osborn AJ, Matsumoto K, Davis RL (2003) Spatiotemporal rescue of memory dysfunction in Drosophila. Science 302:1765-1768.

McGuire SE, Deshazer M, Davis RL (2005) Thirty years of olfactory learning and memory research in Drosophila melanogaster. Prog Neurobiol 76:328-347.

Moita MA, Lamprecht R, Nader K, LeDoux JE (2002) A-kinase anchoring proteins in amygdala are involved in auditory fear memory. Nat Neurosci 5:837-838.

Muller U, Carew TJ (1998) Serotonin induces temporally and mechanistically distinct phases of persistent PKA activity in Aplysia sensory neurons. Neuron 21:1423-1434.

Park SK, Sedore SA, Cronmiller C, Hirsh J (2000) Type II cAMP-dependent protein kinase-deficient Drosophila are viable but show developmental, circadian, and drug response phenotypes. J Biol Chem 275:20588-20596.

Quinn WG, Dudai Y (1976) Memory phases in Drosophila. Nature 262:576-577.

Reisner KJ, Shobe JL, Carew TJ (2006) Molecular nodes in memory processing: insights from Aplysia. Cell Mol Life Sci 63:963-974.

Riemensperger T, Voller T, Stock P, Buchner E, Fiala A (2005) Punishment prediction by dopaminergic neurons in Drosophila. Curr Biol 15:1953-1960.

Schwaerzel M, Heisenberg M, Zars T (2002) Extinction antagonizes olfactory memory at the subcellular level. Neuron 35:951-960.

Schwaerzel M, Monastirioti M, Scholz H, Friggi-Grelin F, Birman S, Heisenberg M (2003) Dopamine and octopamine differentiate between aversive and appetitive olfactory memories in Drosophila. J Neurosci 23:10495-10502.

Sherff CM, Carew TJ (2004) Parallel somatic and synaptic processing in the induction of intermediate-term and long-term synaptic facilitation in Aplysia. Proc Natl Acad Sci USA 101:7463-7468.

Skoulakis EM, Grammenoudi S (2006) Dunces and da Vincis: the genetics of learning and memory in Drosophila. Cell Mol Life Sci 63:975-988.

Tempel BL, Bonini N, Dawson DR, Quinn WG (1983) Reward learning in normal and mutant Drosophila. Proc Natl Acad Sci USA 80:1482-1486.

Wong W, Scott JD (2004) AKAP signalling complexes: focal points in space and time. Nat Rev Mol Cell Biol 5:959-970.

Yu D, Keene AC, Srivatsan A, Waddell S, Davis RL (2005) Drosophila DPM neurons form a delayed and branch-specific memory trace after olfactory classical conditioning. Cell 123:945-957.

Zars T, Fischer M, Schulz R, Heisenberg M (2000) Localization of a shortterm memory in Drosophila. Science 288:672-675. 